\title{
Role of Toll-Like Receptors and Inflammation in Adrenal Gland Insufficiency
}

\author{
Waldemar Kanczkowski ${ }^{a} \quad$ Kai Zacharowski $^{b}$ Stefan R. Bornstein ${ }^{a}$ \\ a Department of Medicine III, Carl Gustav Carus University Hospital, Dresden, and b Clinic of Anaesthesiology, \\ Intensive Care Medicine and Pain Therapy, Frankfurt, Germany
}

\section{Key Words}

Adrenal damage $\cdot$ Pro-inflammatory cytokines $\cdot$ Adrenal hemorrhage $\cdot$ Pattern recognition receptors $\cdot$ Adrenalitis

\begin{abstract}
Adrenal gland insufficiency - the clinical manifestation of deficient production or action of adrenal steroids - is a lifethreatening disorder. Among many factors which can predispose to primary adrenal failure, an autoimmune adrenalitis and infectious agents play a major role. The initial host defense against bacterial infections is executed primarily by the pattern recognition receptors, e.g. Toll-like receptors (TLRs), expressed in cells from the innate immune system. Upon activation, TLRs have been found to regulate various levels of innate and adaptive immunity as well as control tissue inflammation. TLRs are implicated in adrenal cell turnover and steroidogenesis during inflammation. Therefore, TLRs play a crucial role in the activation of adrenal inflammation mediating adrenal gland dysfunction during septicemia.

Copyright $\odot 2010$ S. Karger AG, Base
\end{abstract}

\section{Introduction}

Adrenal gland insufficiency - the clinical manifestation or deficient production of action of glucocorticoids, mineralocorticoids and sex hormones - is a life-threatening disorder that requires prompt clinical management. Insufficiency in adrenal gland steroid production may result from either primary adrenal failure including organ damage or secondary adrenal diseases due to impairment of the hypothalamic-pituitary axis [1].

Symptoms of primary adrenal gland insufficiency as first described in 1855 by Thomas Addison are weakness, fatigue, anorexia, and abdominal pain, with orthostatic hypotension, salt craving, and characteristic hyperpigmentation of the skin. Although the prevalence of socalled Addison's disease (AD) in the general population is low, ranging from 60 up to 117 cases per million, the number of newly diagnosed cases increases annually. Because the full manifestation of the AD occurs when $90 \%$ of the adrenocortical cells are destroyed, the real number of affected individuals may be underestimated. The main cause of the primary adrenal gland failure, besides of genetic factors, is inflammation [2].

Toll-like receptors (TLRs) are critical components of innate immune system. They play an essential role in host defense by eliciting rapid inflammatory reactions and or-

Waldemar Kanczkowski, Department of Medicine III

Carl Gustav Carus Medical School, University of Technology

Fetscherstrasse 74, DE-01307 Dresden (Germany)

Tel. +49351 458 6606, Fax +49351458 6336

E-Mail waldemar.kanczkowski@uniklinikum-dresden.de 
chestrating adaptive innate response to microbial infection. When activated, e.g. during infections, TLRs induce pro-inflammatory cytokine and chemokine synthesis which mediate a rapid recruitment of the leukocytes into the infected tissues. In order to eliminate pathogens, leukocytes release toxic components of their granules including reactive oxygen species (ROS), nitrogen species or, e.g., proteinases. When the acute inflammatory response fails to eliminate the pathogen, or in the case of autoimmune reaction, the inflammatory process persists and results in collateral damage to the host tissues [3].

This article describes the particular role that inflammation plays in adrenal gland insufficiency with a special focus on TLRs.

\section{Adrenal Gland Insufficiency Related to Inflammation}

Among many factors known to affect the adrenal gland such as hereditary disorders, drugs and certain diseases, inflammation plays a special role in the pathogenesis of the adrenal gland. In particular, patients with high risk of developing adrenal gland failure are those with multi-organ autoimmune diseases associated with polyendocrinopathy syndrome (APS) type 1 and type 2, persons with tuberculosis or AIDS-associated cytomegalovirus (CMV) infection and finally critically ill patients with septic shock [1].

In developed countries, autoimmune $\mathrm{AD}$ is a main cause of primary adrenocortical failure, accounting for nearly $80 \%$ of the cases. Clinically, AD often occurs in association with other organ-specific autoimmune disorders such as Hashimoto's thyroid disease, Graves' disease or autoimmune gastritis that are known as APS type 1 and type 2. Although the genetic background and etiology of these syndromes are remarkably different, they both result in a similar autoimmune destruction of the adrenal cortex. The initiation of the pathogenesis of both syndromes is due to a mutation in one of two genes, namely AIRE (autoimmune regulator) for APS1 or CTLA4 (cytotoxic T lymphocyte-associated antigen 4) for APS2. The prevalence of autoreactive antibodies in $\mathrm{AD}$ patients is estimated as $50-90 \%$. The latter antibodies react with P450 enzymes involved in steroid synthesis, 21-hydroxylase (P450c21), 17 $\alpha$-hydroxylase (P450c17) and cholesterol side chain-cleaving enzyme (P450scc). Because P450 enzymes are located within mitochondria, the question is whether auto-antibodies are the cause or the consequence of adrenal destruction. Although the mechanism of autoimmune $\mathrm{AD}$ is not clear yet, the strong association with an inflammatory process has been documented. Therefore, several components of the immune system, including $\mathrm{T}$ cells, autoantibody and complement, are involved in the pathogenesis of adrenocortical destruction. In early stages of the $\mathrm{AD}$ disease the adrenal glands may be enlarged with extensive lymphocytic infiltration. When disease progresses, the adrenal gland becomes atrophic and a strong mononuclear infiltration (mainly lymphocytes but also plasma cells and macrophages) is found [2].

In developing countries and in the case of the immunocompromised patients, infections persist as a main cause of the primary adrenal gland dysfunction. Adrenalitis caused either by tuberculosis or CMV infection is often characterized by strong lymphocytic infiltration (mostly $\mathrm{CD}^{+} \mathrm{T}$ cells) as well as upregulation of many proinflammatory cytokines. Studies using modern diagnostic methods such as computer tomography (CT) revealed that during active tuberculosis, patients usually develop bilateral adrenal gland enlargement with or without calcification. Postmortem histological examination of infected adrenal slices reveals usually a tissue necrosis and mixed inflammatory infiltrate [4]. Considering that almost 2 billion people worldwide are infected with $M y$ cobacterium tuberculosis, a pathogen causing tuberculosis, the chances that some of them will develop adrenal failure during various forms of immune deficiency are high. Similarly to tuberculosis, CMV-induced adrenalitis is also common in patients with AIDS and is usually characterized by focal necrosis and inflammation of the adrenal gland [5].

Adrenal gland impairment is often found in critically ill patients with systemic inflammatory response syndrome and also as a consequence of septic shock. In these patients adrenal gland failure may either be characterized by inadequate glucocorticoid activity in relation to the severity of the patient's illness or manifests itself as a pathogen-induced adrenal hemorrhage. Since the first syndrome is often related to overt cytokine production and dysregulation of immunoadrenal cross talk, the second is frequently manifested as Waterhouse-Friderichsen syndrome and can be directly induced by pathogens. Symptoms of the Waterhouse-Friderichsen syndrome are sudden onset, short course, fever, cyanosis, patching of the skin and mucous membranes, collapse and coma as a result of bilateral adrenal hemorrhage. Despite being mostly associated with meningococcemia, adrenal hemorrhage may also occur after infection with other pathogens including staphylococci [6]. 

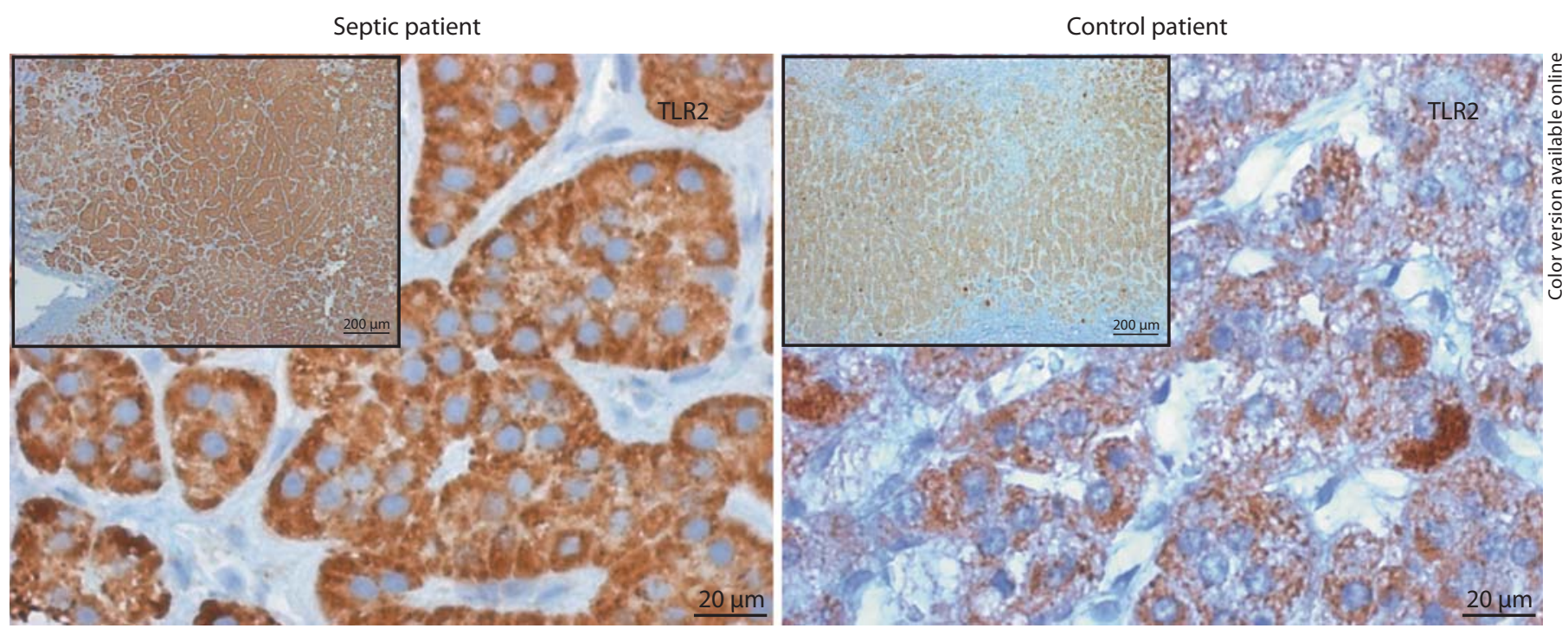

Fig. 1. TLR upregulation in the human adrenal gland during sepsis. Immunohistochemical staining results of human adrenal section showing TLR2 expression of septic adrenal glands (septic patient) and in normal adrenal gland (control patient). The signal was visualized using avidin-biotin labeling and 3,3'-diaminobenzidine. Scale bars are inserted into the right corner and represent $20 \mu \mathrm{m}$ for main picture and $200 \mu \mathrm{m}$ for low magnification picture situated at the left upper corner.

\section{Role of TLRs in Adrenal Gland Insufficiency and Damage}

TLRs are type I transmembrane pattern recognition receptors which are known to regulate various aspects of innate immunity including an inflammatory reaction related to tissue damage or infections. In order to perform their functions, TLRs are located mainly at the cellular membrane but also in the intracellular compartments where they sense various conserved pathogen-associated molecules. The fine examples of TLRs ligands are Gramnegative bacterial endotoxins such as lipopolysaccharide (LPS) sensed by TLR4, or Gram-positive bacterial lipoproteins, e.g. lipoteichonic acid detected by TLR2. Due to their specific localization, TLRs present in the intracellular compartments recognize microbial patterns such as bacterial or viral DNA (TLR9) or RNA (TLR3 and TLR8). To date, 13 TLRs have been found in mammals and activation of all of them induces strong proinflammatory cytokine responses. Due to their ability to initiate an overt inflammation, TLRs are often associated with destruction of various tissues, e.g. during septic shock. Using TLR-deficient animals or TLR antagonists, a direct correlation between hyperactivation of certain TLR and pathogenesis of several diseases including a stroke, dia- betes mellitus, obesity, cardiovascular diseases or autoimmune Hashimoto's disease has been found [3].

TLRs may be involved in the pathogenesis of adrenal gland in many ways. First, during acute or chronic bacterial or viral infections, a strong systemic and local cytokine response is evoked via TLRs expressed in the immune cells, resulting in the alterations of an immunoadrenal cross talk. Furthermore, bacterial and viral infections have been documented to directly impair adrenal gland steroidogenesis, e.g. during adenoviral infection [7]. Moreover, a direct binding of LPS to the surface of adrenocortical cells has been shown by flow-cytometry and was associated with decreased basal and ACTH-induced cortisol production. Further experiments have demonstrated an increased production of ROS which may be the mechanism of the latter observation [8]. Recent data obtained in our laboratory suggest that the negative effect of LPS on adrenocortical cell steroidogenesis could be mediated via TLR4 expressed in steroid-producing cells. Furthermore, we have demonstrated that adrenocortical cells also express other members of the TLR family, which sensitize these cells to pathogenic action [9]. Moreover, we found that TLR2, TLR4 or TLR9 ligands when administered to mice induce a strong systemic and local inflammation of adrenal gland with upregulation of 
the major proinflammatory cytokines such as IL-1, IL-6 and TNF- $\alpha$ [10]. Besides the strong induction of cytokines [9], TLR ligands have been found to directly cause adrenocortical cell apoptosis as well as promote adrenal gland hemorrhage in animal models [11]. The induction of adrenal inflammation is often paralleled with an upregulation of TLR expression, both in vivo and in vitro. A strong upregulation of TLR2 expression can be shown in the adrenal glands of septic patients that directly links adrenal gland damage with TLR overexpression (fig. 1). Although not yet demonstrated in adrenal gland, overstimulation of TLR3 in thyroid gland results in the inflammation and initiation of an autoimmune thyroiditis [12]. Surprisingly, unlike in other organs in which deficiency of one of the TLRs was shown to prevent or limit organ damage during inflammation, e.g. in the brain, TLR 2 and TLR4 deficiency in adrenal gland results in an impaired adrenal glucocorticoid stress response. Moreover, TLR deficiency results in an impairment of the ul- trastructure of the adrenocortical cells indicating a novel role of TLR in the adrenal gland apart from the regulation of the immunoadrenal cross talk [10].

Summarizing, inflammation resulted either from an autoimmune process or, being a part of antimicrobial defense, is associated with adrenal gland insufficiency. The hyperactivation of TLRs in both immune and adrenocortical cells is involved in the pathogenesis of the adrenal gland. Therefore, a development of new treatments and drugs which can antagonize TLR signaling is expected in the future. Results obtained from TLR-deficient mice, however, clearly suggest that a careful monitoring of adrenal gland function during such therapy is necessary.

\section{Acknowledgment}

This work was supported by a grant from the 'Deutsche Forschungsgemeinschaft' to S.R.B. (BO 1141-8-2).

\section{References}

1 Bornstein SR: Predisposing factors for adrenal insufficiency. N Engl J Med 2009;360: 2328-2339.

2 Martin MP, Roep BO, Smit JW: Autoimmunity in Addison's disease. Neth J Med 2002; 60:269-275.

3 Gay NJ, Gangloff M: Structure and function of Toll receptors and their ligands. Annu Rev Biochem 2007;76:141-165.

4 Wang L, Yang J: Tuberculous Addison's disease mimics malignancy in FDG-PET images. Intern Med 2008;47:1755-1756.

5 Price P, Olver SD, Silich M, et al: Adrenalitis and the adrenocortical response of resistant and susceptible mice to acute murine cytomegalovirus infection. Eur J Clin Invest 1996;26:811-819.
6 Hamilton D, Harris MD, Foweraker J, et al: Waterhouse-Friderichsen syndrome as a result of non-meningococcal infection. J Clin Pathol 2004;57:208-209.

7 Alesci S, Ramsey WJ, Bornstein SR, et al: Adenoviral vectors can impair adrenocortical steroidogenesis: clinical implications for natural infections and gene therapy. Proc Natl Acad Sci USA 2002;99:7484-7489.

8 Garcia R, Enriquez de SA, Portoles MT: Calcium and reactive oxygen species as messengers in endotoxin action on adrenocortical cells. Biochim Biophys Acta 1999;14541464.

9 Kanczkowski W, Zacharowski K, Wirth MP, et al: Differential expression and action of Toll-like receptors in human adrenocortical cells. Mol Cell Endocrinol 2009;300:57-65.
10 Zacharowski K, Zacharowski PA, Koch A, et al: Toll-like receptor 4 plays a crucial role in the immune-adrenal response to systemic inflammatory response syndrome. Proc Natl Acad Sci USA 2006;103:6392-6397.

11 Levin J, Cluff LE: Endotoxemia and adrenal hemorrhage: a mechanism for the Waterhouse-Friderichsen syndrome. J Exp Med 1965;121:247-260.

12 Harii N, Lewis CJ, Vasko V, et al: Thyrocytes express a functional toll-like receptor 3: overexpression can be induced by viral infection and reversed by phenylmethimazole and is associated with Hashimoto's autoimmune thyroiditis. Mol Endocrinol 2005; 19 : 1231-1250. 